\title{
Towards a Compact Fiber Laser for Multimodal Imaging
}

\author{
Bai Nie, Ilyas Saytashev and Marcos Dantus
}

\begin{abstract}
We report on multimodal depth-resolved imaging of unstained living Drosophila Melanogaster larva using sub-50 fs pulses centered at $1060 \mathrm{~nm}$ wavelength. Both second harmonic and third harmonic generation imaging modalities are demonstrated.
\end{abstract}

\section{Introduction}

Due to the benefits of high contrast ratio, sub-micrometer resolution and depth resolved imaging multiphoton microscopy has been proven to be a powerful tool for studying living tissues [1, 2]. Especially for second harmonic generation (SHG) or third harmonic generation (THG) microscopy, no sample labeling is needed, which makes those methods preferable for non-invasive in vivo tissue imaging. In addition, SHG and THG provide complementary information due to their different optical-response mechanism. For both SHG and THG imaging, ultrashort laser pulses are preferred to achieve good multiphoton efficiency. It is found that SHG or THG efficiency is inversely proportional to the pulse duration or pulse duration square, respectively [3-6]. For clinical use, a compact and environmentally stable laser is need. In the past decade, fiber lasers have emerged as ideal ultrafast light sources [7]. Here an $\mathrm{Yb}$ fiber oscillator [8], capable of delivering pulses as short as $\sim 50 \mathrm{fs}$ at $1,060 \mathrm{~nm}$ central wavelength, is tested for multiphoton microscopy imaging. The capability of this laser for multiphoton microscopy is evaluated with different samples including prepared slides with stained mouse kidney and mouse intestine sections and unstained living whole Drosophila Melanogaster larva.

\footnotetext{
B. Nie $\cdot$ M. Dantus $(\varangle)$

Department of Physics and Astronomy, Michigan State University, East Lansing, MI 48824, USA

e-mail: dantus@msu.edu

I. Saytashev - M. Dantus

Department of Chemistry, Michigan State University, East Lansing, MI 48824, USA

(C) Springer International Publishing Switzerland 2015

K. Yamanouchi et al. (eds.), Ultrafast Phenomena XIX,

Springer Proceedings in Physics 162, DOI 10.1007/978-3-319-13242-6_182
} 
Images generated by different modalities such as two-photon excited fluorescence (TPEF), SHG and THG are compared. Depth scan of SHG and THG is conducted and reconstructed 3D images are shown.

\section{Experimental}

An Yb fiber oscillator is operated at $43 \mathrm{MHz}$ with average power up to $400 \mathrm{~mW}$. This laser is based on an all-normal dispersion cavity and is similar to the design of the laser described in [8]. The output laser beam is guided through a 4-f folded pulse shaper (MIIPS Box 640, Biophotonic Solutions), which is used to compensate second order and higher phase distortions to deliver transform limited pulses at the focal plane. Output from the pulse shaper is directed to a laser-scanning multiphoton microscope. The laser beam is scanned by a galvanometer mirrors (QuantumDrive-1500, Nutfield Technology, Inc.) and coupled into a water-immersed objective (Zeiss LD C-APOCHROMAT 40x/1.1). The generated SHG and TPEF emissions from samples are collected in the Epi direction, being filtered out using a dichroic mirror (700DCSPXR, Chroma Technology Corp.) and a short-pass emission filter (ET680-SP-2P8, Chroma Technology Corp.). A photomultiplier (PMT, HC20-05MOD, Hamamatsu) is used to collect the SHG/TPEF signal. THG, which is primarily generated in the forward direction, is collected by a UV compatible objective (ReflX NT59-886, NA 0.28, Edmund Optics). The THG signal is also separated from the excitation light by a $400 \mathrm{~nm}$ short pass filter and detected by a PMT (H10720-210, Hamamatsu) whose signal is amplified (SRS445, Stanford Research Systems). The focal plane is moved to different layers using a step motor capable of making precisely controlled $2 \mu \mathrm{m}$ height steps. All the SHG or THG images are then incorporated into a 3-D image.

\section{Results and Discussion}

Excitation laser pulses are compressed to about $50 \mathrm{fs}$ at the focal plane of objective using the MIIPS enabled pulse shaper. To calibrate the microscope, two stained commercial samples (mouse kidney and mouse intestine, Molecular Probes) that have uniform thickness are imaged. For these two samples, the signal detected in Epi direction is mainly from two- or three-photon excited fluorescence. On the forward direction, mainly THG/three-photon excited fluorescence signal is detected. By combining the signal from Epi and forward directions, it is clearly seen that they provide complementary information for each other (see Fig. 1).

Beyond imaging pre-labeled samples, depth-resolved imaging of unstained live tissue is of greater importance. In a previous report [3], we demonstrated a fiber laser delivering $30 \mathrm{fs}$ pulses used for multiphoton imaging of living tissues. However, the low pulse energy (about $1 \mathrm{~nJ}$ ) limited the imaging depth capability. 


\begin{tabular}{|l|lll|}
\hline Layout: T1 Standard Unicode & Book ID: 318422_1_En & Book ISBN: 978-3-319-13241-9 \\
Chapter No.: 182 & Date: $13-12-2014$ & Time: 11:17 am & Page: $\mathbf{3 / 4}$ \\
\hline
\end{tabular}
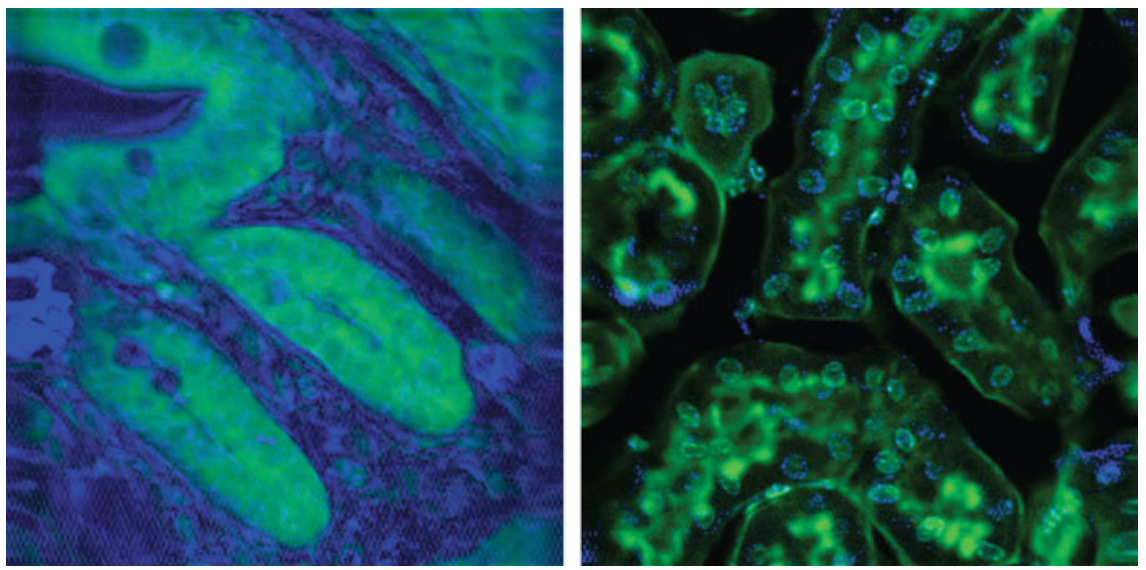

Fig. 1 Composition of TPEF (green, false color) and THG (blue, false color) imaging of mouse intestine (left) and mouse kidney (right), $150 \mu \mathrm{m} \times 150 \mu \mathrm{m}$ area represented

The laser used in this work provides 10 times greater pulse energy and only slightly longer pulse duration. Depth resolved images of third instar Drosophila larva are shown in Fig. 2. The THG 3D image shows many more structures, for example the adipose tissue in the lower left corner, and less scattering than the SHG. The total scanned depth is about $90 \mu \mathrm{m}$.

The shorter pulse durations achieved by the laser greatly enhance two- and threephoton induced modalities in both stained and unstained living tissues.

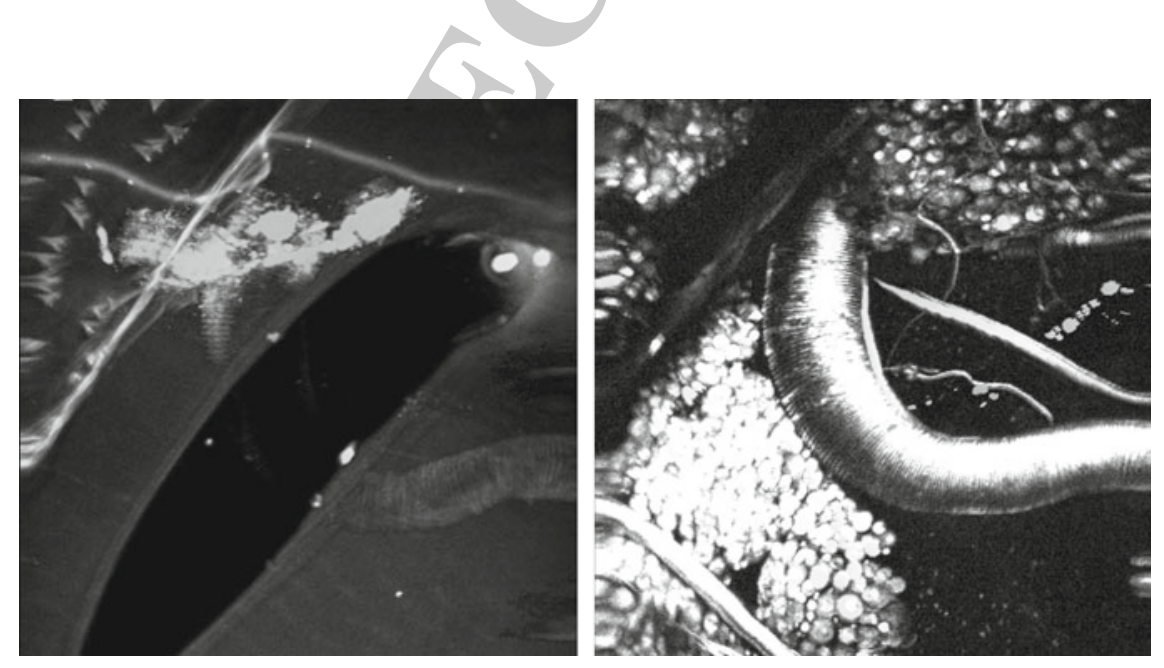

Fig. 2 Projection of 3-D images at $0^{\circ}$ angle for SHG (left) and THG (right) microscopy of the third instar D. Melanogaster larva. Images are of the same $150 \mu \mathrm{m} \times 150 \mu \mathrm{m}$ region centered at the trachea, but different contrast mechanisms highlight different organs 


\section{References}

1. W. Denk et. al, "2-Photon Laser Scanning Fluorescence Microscopy," Science 248, 73-76 (1990).

2. W. R. Zipfel et. al, "Nonlinear magic: multiphoton microscopy in the biosciences," Nature biotechnology 21, 1369-1377 (2003).

3. B. Nie et. al, "Multimodal microscopy with sub-30 fs Yb fiber laser oscillator," Biomed Opt Express 3, 1750-1756 (2012).

4. P. Xi et. al, "Two-photon imaging using adaptive phase compensated ultrashort laser pulses," J Biomed Opt 14 (2009).

5. P. Xi et. al, "Greater signal, increased depth, and less photobleaching in two-photon microscopy with 10 fs pulses," Opt Commun 281, 1841-1849 (2008).

6. A. C. Millard et. al, "Third-harmonic generation microscopy by use of a compact, femtosecond fiber laser source," Appl Optics 38, 7393-7397 (1999).

7. C. Xu, and F. W. Wise, "Recent advances in fibre lasers for nonlinear microscopy," Nature Photonics 7, 875-882 (2013).

8. B. Nie et. al, "Generation of 42-fs and 10-nJ pulses from a fiber laser with self-similar evolution in the gain segment," Opt Express 19, 12074-12080 (2011). 